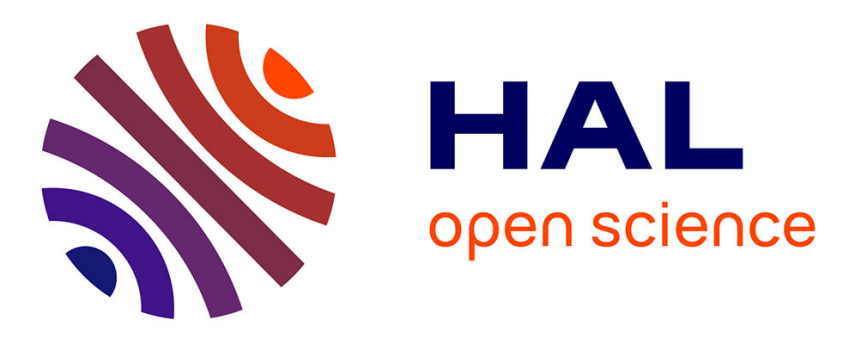

\title{
Thermo-elliptical instability in a rotating cylindrical shell
}

\author{
Michael Le Bars, Stéphane Le Dizès
}

\section{To cite this version:}

Michael Le Bars, Stéphane Le Dizès. Thermo-elliptical instability in a rotating cylindrical shell. Journal of Fluid Mechanics, 2006, 563, pp.189-198. hal-00084046

\section{HAL Id: hal-00084046 https://hal.science/hal-00084046}

Submitted on 5 Jul 2006

HAL is a multi-disciplinary open access archive for the deposit and dissemination of scientific research documents, whether they are published or not. The documents may come from teaching and research institutions in France or abroad, or from public or private research centers.
L'archive ouverte pluridisciplinaire HAL, est destinée au dépôt et à la diffusion de documents scientifiques de niveau recherche, publiés ou non, émanant des établissements d'enseignement et de recherche français ou étrangers, des laboratoires publics ou privés. 


\title{
Thermo-elliptical instability in a rotating cylindrical shell
}

\author{
By M. LE BARS AND S. LE DIZÈS \\ Institut de Recherche sur les Phénomènes Hors Équilibre - CNRS UMR 6594 \\ 49, rue F. Joliot Curie, B.P. 146, F-13384 Marseille Cedex 13, France
}

(Received 5 July 2006)

The linear stability of a rotating flow in an elliptically deformed cylindrical shell with an imposed radial temperature contrast is studied using local and global approaches. We demonstrate that (i) a stabilising temperature profile can either increase or decrease the growth rate of the elliptical instability depending on the selected mode and on the strength of the radial buoyancy force; (ii) when the temperature profile is destabilising, the elliptical instability coexists with $2 \mathrm{D}$ convective instabilities at relatively small values of the Rayleigh number, the fastest growing mode depending on the relative values of the Rayleigh number and of the eccentricity; (iii) the elliptical instability totally disappears for larger values of the Rayleigh number. We argue that thermal effects have to be taken into account when looking for the occurrence and influence of inertial instabilities in geophysical and astrophysical systems, especially in planetary cores.

\section{Introduction}

The elliptical instability corresponds to the 3D destabilisation of $2 \mathrm{D}$ flows with elliptical streamlines. It comes from the parametric resonance of two inertial waves of an undistorted circular flow induced by an underlying strain field (see for instance the review by Kerswell 2002). Most studies of this mechanism have been motivated by applications in turbulence and vortex dynamics, where it is seen as a generic source of intermittency and break-up (e.g. Pierrehumbert 1986; Bayly 1986). But elliptical instabilities also arise in geophysical and astrophysical configurations where rotating fluid bodies are tidally deformed. For instance, their presence has been suggested (i) in binary stars and accretion disks, where they could participate in the energy and angular momentum exchanges between neighbouring systems (e.g. Lubow et al. 1993), and (ii) in planetary cores, where they could participate in the generation or induction of a magnetic field (e.g. Aldridge et al. 1997; Kerswell \& Malkus 1998; Lacaze et al. 2006). In all these natural systems however, thermal effects are also of fundamental importance and leads to various configurations, from stratification to turbulent convection. Results from purely hydrodynamical studies must thus be used with cautions and it appears necessary to investigate how inertial waves and the resonance mechanism leading to the elliptical instability are affected by temperature differences. In particular, one would like to answer the following three questions: (i) how is the growth rate of the elliptical instability modified by thermal effects? (ii) Could the elliptical instability persist in the presence of convection and modify its organisation? (iii) What is the predominant mechanism at the planetary scale?

In this paper, we study analytically the linear stability of a rotating flow in an elliptically deformed cylindrical shell with an imposed radial temperature contrast. Even if this cylindrical geometry is not directly relevant to planetary cores, it appears as a natural, mathematically tractable, first step and has the advantage of being feasible in the 
laboratory. Historically, it has also been used in the first studies of elliptical instabilities and rotating convection (e.g. Malkus 1989; Busse \& Carrigan 1974, respectively), and we expect that it will give us interesting insight into the physics of their interaction.

\section{Basic flow and perturbation equations}

We consider a fluid of density $\rho$, viscosity $\nu$, thermal expansion $\alpha$ and thermal diffusivity $\kappa$ in a shell between an inner cylinder of radius $R_{1}$ and temperature $T_{1}$ and an outer cylinder of radius $R_{2}$ and temperature $T_{2}$. The whole system of height $\tilde{H}$ is rotating at a constant angular velocity $\Omega$. In this paper, we mostly focus on a laboratory experiment in progress. There, the rotation rate is large enough for the vertical gravity to remain negligible compared to the centrifugal acceleration: the fluid is subjected to a radial buoyancy force $B(r)=\alpha\left[T(r)-T_{1}\right] \Omega^{2} r$. The temperature $T_{1}$ can be chosen either lower or larger than $T_{2}$ to induce convection or radial stratification, and the circular sections of both cylinders are slightly deformed into ellipses of eccentricity $\varepsilon$ to generate the elliptical instability. Note that this configuration is also relevant to planetary cores, where the radial buoyancy is due to gravity instead of rotation (Busse \& Carrigan 1974): $B(r)=\alpha\left[T(r)-T_{1}\right] g(r)$, with $g(r)$ depending linearly on the radial coordinate $r$.

In the following, the non-dimensionalisation of the Navier-Stokes and temperature equations is made using $\Omega^{-1}$ as a timescale, $R_{2}$ as a length-scale, $T_{2}-T_{1}$ as a temperaturescale and $\rho_{0} R_{2}^{2} \Omega^{2}$ as a pressure scale. Our system is then characterised by the Ekman number Ek $=\nu / \Omega R_{2}^{2}$, the Rayleigh number $\mathrm{Ra}=B\left(R_{2}\right) / \Omega^{2} R_{2}$, the Prandtl number $\operatorname{Pr}=\nu / \kappa$ and the geometrical parameters $\mathrm{F}=R_{1} / R_{2}, \varepsilon$ and $H=\tilde{H} / R_{2}$.

The base state corresponds to an elliptic flow with a purely diffusive temperature profile. The base flow velocity is given in cartesian coordinates by

$$
\mathbf{u}_{b}=(-(1+\varepsilon) y,(1-\varepsilon) x, 0)
$$

with streamlines defined by $\Gamma_{\varepsilon}^{2}=\frac{x^{2}}{A}+y^{2} A=$ const, where $A$ is the ellipticity $A=\sqrt{\frac{1+\varepsilon}{1-\varepsilon}}$. For small eccentricity and diffusive effects (i.e. $\varepsilon<<1$, Ek $\sim O(\varepsilon)$ and $\operatorname{Pr} \sim O(1)$ as will be considered in the following), isotherms are elliptically deformed such that

$$
T_{b}=1-\frac{\ln \left(\Gamma_{\varepsilon}\right)}{\ln (F)}
$$

and the basic flow pressure field is given, with our normalisation, by

$$
\nabla p_{b}=-\left(\mathbf{u}_{b} \cdot \nabla\right) \mathbf{u}_{b}=\left(1-\varepsilon^{2}\right) \mathbf{r} .
$$

Within the Boussinesq approximation, the Navier-Stokes and temperature equations for the velocity, pressure and temperature perturbations $(\mathbf{u}, p, T)$ to the above base flow reduce to

$$
\begin{gathered}
\frac{\partial \mathbf{u}}{\partial t}+\left(\mathbf{u}_{b} \cdot \nabla\right) \mathbf{u}+(\mathbf{u} \cdot \nabla) \mathbf{u}_{b}=-\nabla p+\operatorname{Ra} T \nabla p_{b}+\operatorname{Ek} \nabla^{2} \mathbf{u}, \\
\frac{\partial T}{\partial t}+\left(\mathbf{u}_{b} \cdot \nabla\right) T+(\mathbf{u} \cdot \nabla) T_{b}=\frac{\mathrm{Ek}}{\operatorname{Pr}} \nabla^{2} T \\
\nabla \cdot \mathbf{u}=0,
\end{gathered}
$$

In addition, the boundary conditions impose (i) no radial velocity, no shear and vanishing temperature fluctuations at the inner and outer deformed cylinders and (ii) no axial velocity, no shear and no heat flux at the top and bottom of the shell. The goal of the 
present paper is to obtain information on the normal mode solution of the above system using analytical approaches.

\section{Global approach}

The spatial structure of the unstable modes can be determined using a global approach in the limit of small $\varepsilon$ and small Ek. We first perform an inviscid analysis of the complete geometry following the approach of Waleffe (1990) and Kerswell (1993), then we add viscous and thermal diffusion effects in the small gap limit (i.e. $F \rightarrow 1$ ). Both simplifications have pros and cons. On the one hand, the small gap approximation allows a complete analytical resolution of the problem, but is a priori not adapted to the laboratory and planetary applications we want to study (for instance in the Earth, $F \sim 0.3$ ). On the other hand, neglecting diffusive effects is fully adapted to the study of inertial instabilities, but prevents the determination of a small-scale cut-off, hence of a critical Rayleigh number for convection.

\subsection{Inviscid approximation}

At order 0 in $\varepsilon$, perturbations in a cylinder of height $H$ can be searched in the form of normal modes

$$
(\mathbf{u}, p, T)=\left(u_{0}(r) \cos (l z), v_{0}(r) \cos (l z), w_{0}(r) \sin (l z), p_{0}(r) \cos (l z), T_{0}(r) \cos (l z)\right) e^{i \omega t+i m \theta},
$$

where the axial boundary conditions simply imply $l=n \pi / H, n$ being an integer. For $l \neq 0$, the system (2.4) can then be reduced to a single Bessel equation for the axial velocity amplitude $w_{0}(\mathrm{Ek}=0, \varepsilon=0)$

$$
\begin{gathered}
r \frac{d}{d r}\left(r \frac{d w_{0}}{d r}\right)+\left(k^{2} r^{2}-\tilde{m}^{2}\right) w_{0}=0, \\
\text { with } k^{2}=\frac{4-\tilde{\mathrm{Ra}}-\lambda^{2}}{\lambda^{2}} l^{2} \text { and } \tilde{m}^{2}=m^{2}\left(1+\frac{\tilde{\mathrm{Ra}}}{\lambda^{2}}\right), \operatorname{Re}(\tilde{m}) \geq 0 .
\end{gathered}
$$

Here, $\lambda=\omega+m$ is the mode frequency in the frame rotating with the cylindrical shell and $\tilde{\mathrm{Ra}}=-\mathrm{Ra} / \ln (F)$ is positive for a destabilising temperature profile $\left(T_{1}<T_{2}\right)$. If we enforce the boundary conditions $u_{0}(1)=u_{0}(F)=0$, i.e.

$$
r \frac{d w_{0}}{d r}+\frac{2 m}{\lambda} w_{0}=0 \text { at } r=F, 1,
$$

to the general solution of this equation

$$
w_{0}=C_{1} J_{\tilde{m}}(k r)+C_{2} Y_{\tilde{m}}(k r),
$$

we obtain the dispersion relation between the frequency $\lambda$ (or $\omega$ in the non-rotating frame) and the axial wavenumber $l$ for given values of $m$ and Ra. Note that the system is invariant by changing $l$ in $-l$ so only $l>0$ is considered. For $l=0,(3.2)$ does not apply and the adequate equation is for the radial velocity amplitude $u_{0}$ :

$$
\frac{d^{2} u_{0}}{d r^{2}}+\frac{3}{r} \frac{d u_{0}}{d r}-\frac{m^{2}-1+m^{2} \tilde{\mathrm{Ra}} / \lambda^{2}}{r^{2}} u=0
$$

which admits solutions of the type

$$
u_{0}=C_{1} r^{-1+m \sqrt{1+\tilde{\mathrm{Ra}} / \lambda^{2}}}+C_{2} r^{-1-m \sqrt{1+\tilde{\mathrm{Ra}} / \lambda^{2}}} .
$$



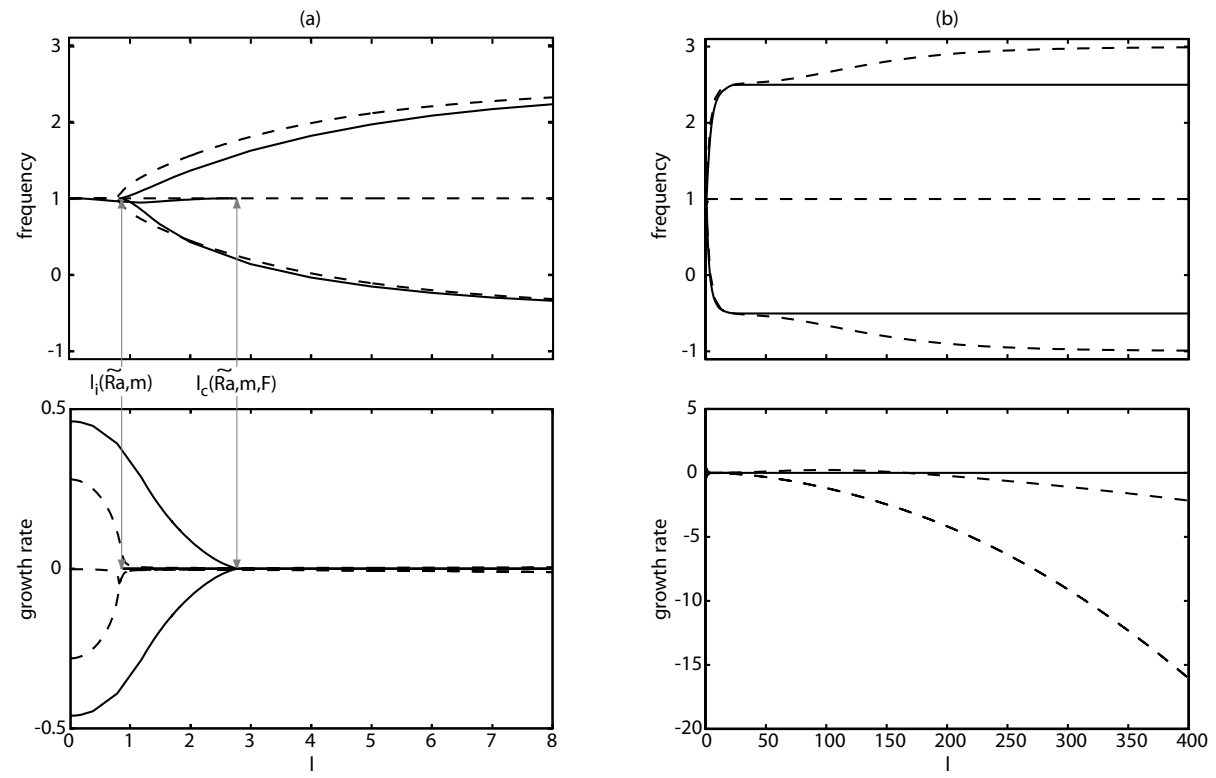

FIGURE 1. Frequency $\operatorname{Re}(\omega)$ and growth rate $-\operatorname{Im}(\omega)$ of the first azimuthal mode $m=-1$ determined from the inviscid analysis (continuous line) and the viscous small gap analysis (dashed line) for $\tilde{R} a=1.66, F=0.3, E k=10^{-4}, \mathrm{Pr}=7$. Inertial modes correspond to the oscillatory neutral modes (non-zero frequency and zero growth rate), whereas convective modes have an almost zero frequency in the rotating frame and a non-zero growth rate.

The boundary conditions $u_{0}(F)=u_{0}(1)=0$ provide an explicit expression for $\lambda$

$$
\lambda^{2}=-\frac{\tilde{\mathrm{Ra}}}{1+\left(\frac{p \pi}{m \ln (F)}\right)^{2}},
$$

where $p$ is a non-zero integer standing for the number of radial structures.

The dispersion relation leads to infinitely many branches discretized by the radial wavenumber $k$. The first of them is shown in figure 1 (a) for $m=-1$ and $\tilde{R a}=1.66$. Results at lower/higher Ra can be deduced qualitatively by shifting the axes system towards the right/left. For $\tilde{\mathrm{Ra}}<4$, neutral inertial waves exist for $l \geq l_{i}(\tilde{\mathrm{Ra}}, m)$. From (3.3), we can show that their frequency in the rotating frame satisfies

$$
-\sqrt{4-\tilde{\mathrm{Ra}}} \leq \lambda \leq \sqrt{4-\tilde{\mathrm{Ra}}}
$$

The threshold wavenumber $l_{i}$ vanishes for $\tilde{\text { Ra }} \leq 0$, and is given by $l_{i}=\sqrt{m^{2} \tilde{\mathrm{Ra}} /(4-\tilde{\mathrm{Ra}})}$ for $\tilde{\mathrm{Ra}}>0$. Then, one stable and one unstable mode appear in the vicinity of $l=0$. These modes are localised near the inner cylinder and can coexist with inertial waves, localised near the outer cylinder. Indeed, they persist for $l \leq l_{c}=\sqrt{m^{2} \tilde{\mathrm{Ra}} /(4-\tilde{\mathrm{Ra}}) F^{2}}$. The maximum growth rate takes place at $l=0$ and is provided by (3.8): it corresponds to the well-known 2D $(w=0)$ z-independent Busse columns at onset of convection (see for instance the review by Busse 2002). This two-dimensional convective mode is dominant for relatively small Ra. For Ra $>4$, the inertial modes disappear and all axial wavenumbers become unstable by convection. The most unstable mode at a given $m$ changes from $l=0$ to $l \rightarrow \infty$ for some R̃a $\sim O(4)$. 

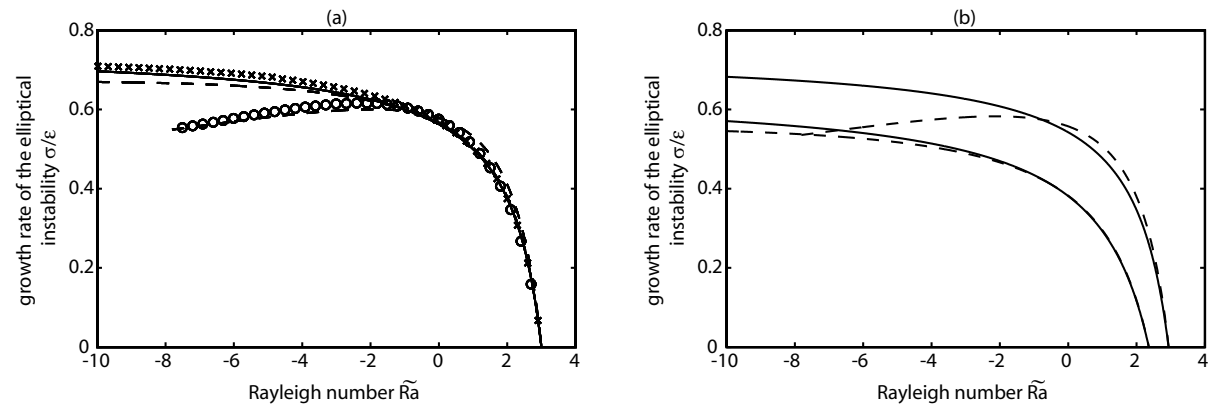

FIGURE 2. Normalised growth rate of the elliptical instability $\sigma$ divided by the eccentricity $\varepsilon$ versus $\tilde{R}$ a for $F=0.3$. Symbols: numerical computations of the global growth rate for the first mode (circles) and the third mode (crosses); dashed lines: small gap growth rate according to the analytical formula (3.17) with $k=\pi /(1-F)$ and $k=3 \pi /(1-F)$ respectively; continuous lines: local growth rate according to the analytical formulas (4.7) and (4.8) with $k=\pi /(1-F)$ and $k=3 \pi /(1-F)$ respectively. (a) Inviscid results and (b) viscous results for $E k=10^{-4}, \operatorname{Pr}=7.0, \varepsilon=0.1$.

In order to describe the elliptical instability in this global framework, mode coupling at the order $\varepsilon$ has to be taken into account. As shown in Moore \& Saffman (1975), the elliptical instability results from the resonant coupling of the strain field due to the elliptical deformation with two inertial modes of azimuthal wavenumbers $m$ and $m+2$ and identical axial wavenumber $l$ and frequency $\omega$. The most unstable resonant configuration corresponds to the coupling between the two azimuthal modes $m=1$ and $m=-1$ at $\omega=0$ with the same radial wavenumber $k$. The elliptical instability growth rate $\sigma$ can then be obtained using Kerswell (1993) analysis: it is reported in figure 2(a) as a function of the Rayleigh number. Note that the resonance of two azimuthal modes $m=1$ and $m=-1$ with $p$ radial structures can only be obtained for

$$
-1-(p \pi / \ln (F))^{2}<\tilde{\mathrm{Ra}}<3 .
$$

Indeed, the axial wavenumber $l$ of the resonant modes increases with Ra and goes to infinity when $\tilde{\mathrm{Ra}} \rightarrow 3$ and to 0 when $\tilde{\mathrm{Ra}} \rightarrow-1-(p \pi / \ln (F))^{2}$. For $\tilde{\mathrm{Ra}}>3$ and $\tilde{\mathrm{Ra}}<-1-$ $(p \pi / \ln (F))^{2}$, no coupling is possible and elliptical instability is expected to disappear. One can notice that a stabilising temperature profile (i.e. $-1-(p \pi / \ln (F))^{2}<\tilde{R a}<0$ ) can either increase or decrease the growth rate of the elliptical instability, depending on the selected resonance. For $0 \leq \tilde{\mathrm{Ra}}<3$, the elliptical instability is in competition with the convective instability, the fastest growing mode depending on the relative value of $\tilde{\mathrm{Ra}}$ and $\varepsilon$.

\subsection{Small gap approximation}

In the small gap limit (i.e. $F \sim 1$ ), one can neglect curvatures effects and take $r \sim 1$ everywhere, the radial dependence only appearing in the radial derivatives. The perturbed equations (2.4) then reduce to a single linear equation of order 8 for the temperature amplitude $T_{0}$ of the normal mode (3.1)

$$
\left[\left(\frac{d^{2}}{d r^{2}}-\left(m^{2}+l^{2}\right)\right) L_{1}^{2} L_{2}-\left(m^{2}+l^{2}\right) \tilde{R a} L_{1}-4(i m \mathrm{Ek}-1)^{2} l^{2} L_{2}\right] T_{0}=0,
$$

with the boundary conditions $T_{0}(1)=T_{0}(F)=0$, where the operator $L_{1}, L_{2}$ are defined by

$$
L_{1}=i \lambda-\operatorname{Ek}\left(\frac{d^{2}}{d r^{2}}-\left(m^{2}+l^{2}\right)\right) ; \quad L_{2}=-i \lambda+\frac{\mathrm{Ek}}{\operatorname{Pr}}\left(\frac{d^{2}}{d r^{2}}-\left(m^{2}+l^{2}\right)\right) .
$$


Solutions are searched in the form $T_{0}(r)=T_{p} \sin (k(r-1))$, where $T_{p}$ is a constant and $k=p \pi /(1-F)$ with $p$ a positive integer. Substituting in (3.11), we obtain the dispersion relation

$$
\begin{aligned}
\lambda^{3} & -i K^{2} \mathrm{Ek}(2+1 / \operatorname{Pr}) \lambda^{2}-\frac{K^{6} \mathrm{Ek}^{2}(1+2 / \mathrm{Pr})+4(i m \mathrm{Ek}-1)^{2} l^{2}-\left(m^{2}+l^{2}\right) \tilde{\mathrm{Ra}}}{K^{2}} \lambda \\
& +i\left(K^{6} \mathrm{Ek}^{3} / \operatorname{Pr}+4(i m \mathrm{Ek}-1)^{2} l^{2} \mathrm{Ek} / \mathrm{Pr}-\left(m^{2}+l^{2}\right) \tilde{\mathrm{RaEk}}\right)=0
\end{aligned}
$$

with $K=\sqrt{k^{2}+l^{2}+m^{2}}$. The dispersion relation is of order 3 in $\lambda=\omega+m$, which means that for each given set of parameters ( $\tilde{\mathrm{Ra}}, \mathrm{Ek}, \operatorname{Pr}, \mathrm{F}, l, m, k), 3$ different modes coexist: a purely diffusive mode that disappears for $\mathrm{Ek}=0$ and two inertial modes.

As shown in figure 1(a), the small gap limit captures well the inertial modes of $m=-1$ for values of $F$ as small as 0.3 . Note however that the unstable convective mode and neutral inertial mode cannot coexist in the small gap limit: they both possess the same sinusoidal profile which does not allow the localisation near one boundary. Besides, the behaviour at large $l$ significantly changes, as seen in figure 1(b): we first recover the inviscid results in the intermediate limit $1<<l^{2}<<1 / \mathrm{Ek}$, but in the limit $l^{2}>>1 / \mathrm{Ek}$, the asymptotic behaviour of the 3 solutions of (3.13) is

$$
\begin{aligned}
\operatorname{Re}(\lambda) & \rightarrow 0 \text { and } \operatorname{Im}(\lambda) \sim l^{2} \frac{\mathrm{Ek}}{\operatorname{Pr}} \text { for the diffusive mode, } \\
\operatorname{Re}(\lambda) & \rightarrow \pm 2 \text { and } \operatorname{Im}(\lambda) \sim l^{2} \text { Ek for the } 2 \text { inertial modes. }
\end{aligned}
$$

In fact, at small wavelength, the diffusive effects completely kill convective effects and we end up with exactly the same modes as in the case $\tilde{R} a=0$. This limit is however not interesting since all the modes as well as the elliptical instability are then damped by viscosity.

The onset of convection can be analysed by considering two-dimensional modes $(l=0)$. Then, the dispersion relation (3.13) admits an unstable solution providing

$$
\mathrm{Ra} \geq \operatorname{Ra}_{c}=\frac{27 \pi^{4}}{4} \frac{\mathrm{Ek}^{2}}{\operatorname{Pr}(1-F)^{3}} .
$$

With the standard definition of the Rayleigh number using the ratio of buoyancy with diffusion (rather than inertia), this means

$$
\frac{\alpha g\left(T_{2}-T_{1}\right)\left(R_{2}-R_{1}\right)^{3}}{\kappa \nu} \geq \frac{27 \pi^{4}}{4}
$$

as already found for the onset of Rayleigh-Bénard convection as well as for the onset of convection in the rapidly rotating cylindrical shell (Busse 1970).

The viscous growth rate of the elliptical instability $\sigma$ can be calculated explicitly, assuming $\mathrm{Ek} \sim O(\varepsilon)$ and $\operatorname{Pr} \sim O(1)$ :

$$
\begin{aligned}
\sigma= & \frac{9-3 \tilde{\mathrm{Ra}}+\frac{36-18 \tilde{\mathrm{Ra}}+2 \tilde{\mathrm{Ra}}^{2}}{k^{2}}+\frac{18-12 \tilde{\mathrm{Ra}}+2 \tilde{\mathrm{Ra}}^{2}}{k^{4}}}{16-4 \tilde{\mathrm{Ra}}+\frac{54-36 \tilde{\mathrm{Ra}}+6 \tilde{\mathrm{Ra}}^{2}}{k^{2}}+\frac{18-30 \tilde{\mathrm{Ra}}+14 \tilde{\mathrm{Ra}}^{2}-2 \tilde{\mathrm{Ra}}^{3}}{k^{4}}} \varepsilon \\
& -k^{2} \mathrm{Ek} \frac{8-\tilde{\mathrm{Ra}}(1+1 / \mathrm{Pr})+\frac{11-2 \tilde{\mathrm{Ra}}-\tilde{\mathrm{Ra}}(4-\tilde{\mathrm{Ra}}) / \mathrm{Pr}}{k^{2}}+\frac{3-\tilde{\mathrm{Ra}}-\tilde{\mathrm{Ra}}(3-\tilde{\mathrm{Ra}}) / \mathrm{Pr}}{k^{4}}}{8-2 \tilde{\mathrm{Ra}}+\frac{3-4 \tilde{\mathrm{Ra}}+\tilde{\mathrm{Ra}}^{2}}{k^{2}}}
\end{aligned}
$$

As shown in figure 2(a), this formula provides a good approximation of the numerical results, even for $F=0.3$. Regarding diffusion, one can notice that the first corrective term at large $k$ is proportional to $k^{2} \mathrm{Ek}$, as in the non-thermal case (see Eloy et al. 

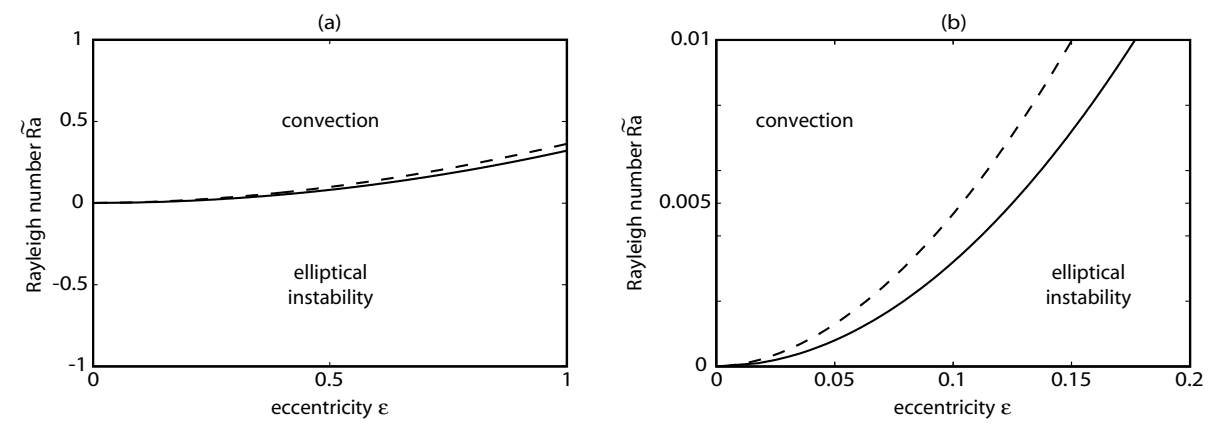

FIGURE 3. Most dangerous instability in the eccentricity $\varepsilon$ - Rayleigh number $\tilde{R} a$ plane for an adjusted cylinder with $F=0.3$. Dashed line: small gap viscous results with $E k=10^{-4}, \operatorname{Pr}=7, k=\pi /(1-F)$ and continuous line: local inviscid results. (b) shows a zoom of (a) in the parameter range that will be explored by the experimental device.

2003). But surprisingly, diffusive effects are expected to have a destabilising influence on the elliptical instability at positive R̃a and relatively small Pr. Indeed, convection has a general stabilising influence on the elliptical instability. Since increasing the thermal diffusion leads to decreasing the intensity of convection, one can expect that for a given value of the Rayleigh number, the growth rate of the elliptical instability at small Pr will be relatively larger.

Finally, the competition between the elliptical and the convective instabilities can also be investigated explicitly, supposing that the most dangerous mode will be the fastest growing one: it is shown as a function of $\tilde{\mathrm{Ra}}$ and $\varepsilon$ in figure 3 for the first unstable mode (i.e. $p=1$ ).

\section{Local approach}

The local approach is based on the inviscid short-wavelength Lagrangian theory developed by Bayly (1986); Craik \& Criminale (1986) and generalised by Friedlander \& Vishik (1991); Lifschitz \& Hameiri (1991). In this approach, perturbations are sufficiently localised in order to be advected along flow trajectories defined by

$$
x(t)=r \sqrt{A} \cos (\gamma t), y(t)=\frac{r}{\sqrt{A}} \sin (\gamma t), z(t)=\text { const },
$$

where $r$ is a given constant and $\gamma=\sqrt{1-\varepsilon^{2}}$. Perturbations are searched as local plane waves of the form

$$
(\mathbf{u}(x, y, z, t), p(x, y, z, t), T(x, y, z, t))=(\mathbf{u}(t), p(t), T(t)) e^{i \mathbf{k}(t) \cdot \mathbf{x}} .
$$

with a wavevector $\mathbf{k}$ defined by

$$
\mathbf{k}(t)=k\left(\frac{\sin (a)}{\sqrt{A}} \cos \left(\gamma\left(t-t_{0}\right)\right), \sin (a) \sqrt{A} \sin \left(\gamma\left(t-t_{0}\right)\right), \cos (a)\right),
$$

where $k, a, t_{0}$ are constants, such that the phase of the wave (4.2) is conserved.

The local approach allows a complete viscous analytical resolution of the system. Indeed, when (4.2) and (4.3) are inserted into (2.4), a system of ordinary equations is obtained in the Lagrangian frame for the perturbation amplitude $(\mathbf{u}(t), p(t), T(t))$. When the base flow is undistorted $(\varepsilon=0)$ and inviscid $(\mathrm{Ek}=0),(2.4)$ reduces to a single 
equation for the amplitude of the axial velocity

$$
\frac{d^{2} w_{0}}{d t^{2}}+(4-\tilde{\mathrm{Ra}}) \cos ^{2}(a) w_{0}=0,
$$

where the subscript 0 indicates the order 0 of the perturbative approach in $\varepsilon$. The solution is given by $w_{0}=C_{1} e^{i f t}+C_{2} e^{-i f t}$, where $C_{1}$ and $C_{2}$ are constants and $f$ is given by the dispersion relation

$$
f=\sqrt{4-\tilde{\mathrm{Ra}}} \cos (a) .
$$

According to this dispersion relation, several cases are possible depending on the Rayleigh number Ra. If $\tilde{R a}<4$, the undistorted system is inviscidly stable. However, as shown for instance by Waleffe (1990) and Le Dizès (2000), an elliptical instability is possible in the elliptically deformed system if the frequency $f$ of the plane wave solution matches the frequency of the elliptical forcing in the Lagrangian frame, that is $f= \pm 1$. From (4.5), such a resonance is possible when

$$
\cos (a)=\frac{ \pm 1}{\sqrt{4-\tilde{R a}}},
$$

The growth rate associated with this resonance can be obtained by an asymptotic analysis for small $\varepsilon$ (e.g. Le Dizès 2000). The maximum growth rate is obtained when $\mathbf{k}$ is orthogonal to the base flow velocity (i.e. $t_{0}=0$, see Sipp \& Jacquin 1998) and is found to be given by a simple formula

$$
\sigma=\frac{9-3 \tilde{\mathrm{Ra}}}{16-4 \tilde{\mathrm{Ra}}} \varepsilon .
$$

As shown in figure 2, this fully agrees with the numerical results of the inviscid global approach and with the small gap analytical results (3.17) in the limit $k \rightarrow \infty$ relevant to the local approach. Diffusive effects can be easily taken into account in the limit $\mathrm{Ek} \sim O(\varepsilon)$ and induce a correction

$$
-k^{2} \operatorname{Ek}(8-\tilde{\operatorname{Ra}}(1+1 / \operatorname{Pr})) /(8-2 \tilde{\operatorname{Ra}}),
$$

in agreement with the first corrective term induced by diffusion in the small gap formula (3.17). As Ra increases, the growth rate decreases and the resonant wavector given by (4.6) progressively aligns with the rotation axis. Motions then depend on $z$ only, which implies from the mass conservation equation that $w=0$ : convective effects thus tend to bidimensionalise the perturbed flow (see also Busse 1970), hence killing the 3D elliptical instability. For $\tilde{\mathrm{Ra}}>3$, the perfect resonance is impossible and the elliptical instability disappears, as also seen in the global approach. No lower bound is found for Ra in the local approach, in agreement with (3.10) in the relevant limit $p \rightarrow \infty$.

If $\tilde{R a}>4, f$ given by (4.5) is purely imaginary: the undistorted system thus admits local unstable modes with a maximum growth rate $\sigma=\sqrt{\tilde{\text { Ra }-4}}$ obtained for $\cos (a)=1$. This instability criterion R̃a $>4$ is the direct translation of the well known criterion for centrifugal instability $G^{2}>\Phi$, where $-G^{2}$ is the Brunt-Väisälä frequency and $\Phi$ the Rayleigh discriminant (Eckhoff 1984; Sipp et al. 2005). These unstable modes have to be distinguished from the 2D Busse columns, for which $w_{0}=0$ and all variables are $z$-independent. Such instabilities can be obtained for the local approach by considering local plane wave satisfying $\cos (a)=0$. For these $2 \mathrm{D}$ waves, we immediately write a single equation for the temperature amplitude $T_{0}$

$$
d_{t}^{2} T_{0}+k^{2} \operatorname{Ek}\left(1+\frac{1}{\mathrm{Pr}}\right) d_{t} T_{0}+\left(\frac{k^{4} \mathrm{Ek}^{2}}{\operatorname{Pr}}-\tilde{\operatorname{Ra}}\left(1-\cos ^{2} t_{0}\right)\right) T_{0}=0
$$


from which we deduce the criterion for instability $\tilde{\mathrm{Ra}}>\mathrm{Ra}_{c}=\frac{k^{4} \mathrm{Ek}^{2}}{\operatorname{Pr}}$ and the value of the most unstable growth rate.

The local approach thus leads to the same conclusions as the global approach, but in addition it allows a simpler and fully analytical resolution of the system without using the small gap approximation. In particular, the competition between $2 \mathrm{D}$ convection and 3D elliptical instability can be solved analytically: based on the local inviscid results, we expect convection to be dominant over the elliptical instability as soon as $\tilde{R a} \geq 0.32 \varepsilon^{2}$, as shown in figure 3 .

\section{Conclusion}

In this paper, we have studied by complementary analytical methods the linear stability of a rotating flow in an elliptically deformed cylindrical shell with an imposed radial temperature profile. We have shown that the elliptical instability has a decreasing growth rate with $\tilde{\mathrm{Ra}}$. It is active for $\tilde{\mathrm{Ra}}<3$ and is in competition with the two-dimensional convection instability leading to Busse columns as summarized in figure 3. From the linear study proposed here, we can only expect that the most dangerous mode will be the fastest growing one. Non-linear processes of this competition will be studied in a future laboratory experiment, in the parameter ranges $-10^{-2} \leq \tilde{\mathrm{Ra}} \leq 10^{-2}$ and $0 \leq \varepsilon \leq 0.20$ (see figure 3(b)).

In the bulk of the Earth core, $\tilde{\mathrm{Ra}} \sim O(0.1-10)$ and $\varepsilon \sim O\left(10^{-7}\right)$, thus two-dimensional convection is expected to dominate over elliptical instability: it is indeed supposed to drive motions under the form of mainly axial propagating cells powering the geodynamo (see for instance the review by Busse 2000). However, this does not preclude the presence of elliptical instabilities, either superimposed on the mean temperature profile induced by convection or in the outer stratified part of the core (Kerswell 1993). In Io, the temperature gradient in the core is unsufficient to drive convection (Wienbruch \& Spohn 1995): our study thus suggest that the elliptical instability could be the dominant phenomenon with a growth rate slightly smaller than the usual asymptotic value of $9 / 16 \varepsilon$ (see Kerswell \& Malkus 1998). Note however than the results shown here are adapted to the experimental configuration, where the centrifugal acceleration stands for the radial gravity. In particular, the equipotentials remain circular at first order in $\varepsilon$. One could suggest in planetary applications that tides in the core also deform equipotentials elliptically: in this case, the local approach gives a modified growth rate $\sigma=\frac{9-\tilde{\mathrm{Ra}} / 2}{16-4 \tilde{\mathrm{Ra}}} \varepsilon$, which is a growing function of $\tilde{\mathrm{Ra}}$, as opposed to the previous results. Nevertheless, we also find that the elliptical instability is only possible providing $\tilde{R} a<3$ and that its growth rate remains of order $(0.1-1.0) \varepsilon$ typically.

So as a conclusion, we want to highlight that even if the quantitative results will depend on the chosen model of the planetary core, the physics of the interaction between thermal effects and elliptical instability will remain similar to what is studied here. Thermal effects have thus to be taken into account when studying the occurence in planetary cores of inertial processes such as tidal instabilities, but also such as precession, which similarly involves inertial wave coupling.

\section{REFERENCES}

Aldridge, K., Seyed-Mahmoud, B., Henderson, G. \& van Wijngaarden, W. 1997 Elliptical instability of the Earth's fluid core. Phys. Earth Planet. Int. 103, 365-74.

BAyly, B. J. 1986 Three-dimensional instability of elliptical flow. Phys. Rev. Lett. 57, 2160-63. 
Busse, F. H. 1970 Thermal instabilities in rapidly rotating systems. J. Fluid Mech. 44, 441-460.

Busse, F. H. 2000 Homogeneous dynamos in planetary cores and in the laboratory. Annu. Rev. Fluid Mech. 32, 383-408.

Busse, F. H. 2002 Convective flows in rapidly rotating spheres and their dynamo action. Phys. Fluids 14, 1301-14.

Busse, F. H. \& CARrigan, C. R. 1974 Convection induced by centrifugal buoyancy. J. Fluid Mech. 62, 579-592.

Craik, A. D. D. \& Criminale, W. O. 1986 Evolution of wavelike disturbances in shear flows - a class of exact solutions of the Navier-Stokes equations. Proc. Roy. Soc. A406, 13-26.

ECKHOFF, K. S. 1984 A note on the instability of columnar vortices. J. Fluid Mech. 145, 417-21.

Eloy, C., Le GaL, P. \& LE Dizès, S. 2003 Elliptic and triangular instabilities in rotating cylinders. J. Fluid Mech. 476, 357-388.

FriEdLANDER, S. \& ViShIK, M. 1991 Instability criteria for steady flows of a perfect fluid. Phys. Rev. Lett. 66, 2204-2206.

KeRswell, R. R. 1993 Elliptical instabilities of stratified, hydromagnetic waves. Geophys. Astrophys. Fluid Dyn. 71, 105-143.

Kerswell, R. R. 2002 Elliptical instability. Annual Review of Fluid Mechanics 34, 83-113.

Kerswell, R. R. \& Malkus, W. V. R. 1998 Tidal instability as the source for Io's magnetic signature. Geophys. Res. Lett. 25, 603-6.

Lacaze, L., Herreman, W., Le Bars, M., Le Dizès, S. \& Le Gal, P. 2006 Magnetic field induced by elliptical instability in a rotating spheroid. Geophys. Astrophys. Fluid Dyn., in press.

LE Diż̀s, S. 2000 Three-dimensional instability of a multipolar vortex in a rotating flow. Phys. Fluids 12, 2762-74.

Lifschitz, A. \& HAmeiri, E. 1991 Local stability conditions in fluid dynamics. Phys. Fluids 3, 2644-2651.

Lubow, S. H., Pringle, J. E. \& Kerswell, R. R. 1993 Tidal instability of accretion disks. ApJ 419, 758-67.

Malkus, W. V. R. 1989 An experimental study of the global instabilities due to the tidal (elliptical) distortion of a rotating elastic cylinder. Geophys. Astrophys. Fluid Dyn. 48, 123-34.

Moore, D. W. \& Saffman, P. G. 1975 The instability of a straight vortex filament in a strain field. Proc. R. Soc. Lond. A. 346, 413-425.

Pierrehumbert, R. T. 1986 Universal short-wave instability of two-dimensional eddies in an inviscid fluid. Phys. Rev. Lett. 57, 2157-59.

Sipp, D., FABre, D., Michelin, S. \& Jacquin, L. 2005 Stability of a vortex with a heavy core. J. Fluid Mech. 526, 67-76.

SiPP, D. \& JACQUin, L. 1998 Elliptic instability in two-dimensional flattened Taylor-Green vortices. Phys. Fluids 10, 839-49.

WALEFfe, F. A. 1990 On the three-dimensional instability of strained vortices. Phys. Fluids 2, $76-80$.

Wienbruch, U. \& Spohn, T. 1995 A self-sustained magnetic field on Io? Planet. Space. Sci. 43, 1045-1057. 